\title{
On the use of Slepian Functions for the Reconstruction of the Head-Related Transfer Function on the Sphere
}

\author{
Alice P. Bates ${ }^{1}$, Zubair Khalid ${ }^{2}$ and Rodney A. Kennedy ${ }^{1}$ \\ ${ }^{1}$ Research School of Engineering, College of Engineering and Computer Science, \\ The Australian National University, \\ Canberra, ACT 2601, Australia \\ ${ }^{2}$ Department of Electrical Engineering, SBA School of Science and Engineering, \\ Lahore University of Management Sciences, \\ Lahore 54792, Pakistan \\ Email: alice.bates@anu.edu.au, zubair.khalid@lums.edu.pk, rodney.kennedy@anu.edu.au \\ Work supported by Australian Research Council's Discovery Projects funding scheme (Project no. DP150101011).
}

\begin{abstract}
In this work, we perform an investigation into using the Slepian, rather than the traditionally used spherical harmonic, basis for the reconstruction of the head-related transfer function (HRTF) on the sphere. Measurements of the HRTF are unavailable over the south polar cap which tends to result in large reconstruction errors when using the spherical harmonic basis. While the spherical harmonic basis is well-suited to applications where data is taken over the whole sphere, it is not a natural basis when considering a region on the sphere. The Slepian basis is a set of functions which are optimally concentrated and orthogonal within a region, unlike the spherical harmonic basis. We demonstrate through numerical experiments on randomly generated data and synthetic HRTF measurements that reconstruction of the HRTF in the Slepian basis is significantly more accurate at sample locations, the reconstruction error is up to 11 orders of magnitude smaller, as well as other locations on the sphere, both within and outside of the region where measurements are taken, than in the spherical harmonic basis. We also briefly investigate truncation of the Slepian basis as a means of denoising the HRTF measurements and find that this reduces the reconstruction error. Our analysis suggests that the Slepian basis allows more accurate reconstruction than the spherical harmonic basis.

Index Terms-sampling; unit sphere; Slepian functions; spectral analysis; head-related transfer function (HRTF) measurements.
\end{abstract}

\section{INTRODUCTION}

The head-related transfer function (HRTF) is an acoustic transfer function which measures how sound waves are altered by a listener's head and torso before reaching the eardrum [1]-[3]. HRTFs are unique for an individual; knowledge of a listener's HRTF allows for synthesis of sound which appears to be coming from any chosen direction using binaural signals. HRTF measurements are normally taken in the audible frequency range $([0.2,20] \mathrm{kHz})$ using microphones (or speakers) arranged at a constant distance from a subject on a sphere [4] [6]. The HRTF at a particular frequency and constant distance from the listener is a signal on the sphere. It is therefore common for the HRTF to be reconstruction from a finite number of measurements, where the number of measurements should be as small as possible to minimise acquisition time and cost [7], by expansion in the spherical harmonic basis [1], [2], [4], [8], [9], where spherical harmonics are the archetype set of complete basis functions orthonormal over the sphere.

One of the major issues in HRTF reconstruction is that due to ground reflections and limitations of the sampling apparatus, measurements are unavailable in the south polar cap region (at co-latitudes greater than about $140^{\circ} \approx 0.8 \pi$ radians) [3]-[6]. When samples are unavailable over part of the sphere, large errors occur when reconstructing the HRTF in the spherical harmonic basis [7], [8]. This is because spherical harmonics are not a natural basis for the region; they are not orthogonal over any region of the sphere other than the whole sphere [10]. A similar problem, called the polar gap, occurs in geophysics where measurements are only available in the latitudinal belt (no measurements are available at either the North or South pole) [11], [12]. Slepian functions, another complete set of basis functions on the sphere which are not only orthonormal on the whole sphere but also orthogonal over a given region of the sphere, have been used to solve the polar gap problem with greater reconstruction accuracy than was obtained using spherical harmonics [10].

\section{A. Contributions}

In this work we perform a preliminary investigation into using the Slepian basis, rather than the spherical harmonic basis, to reconstruct HRTFs on the sphere when measurements are unavailable over the south polar cap. In particular, we present a HRTF reconstruction method using the Slepian basis and evaluate this method against reconstruction in the spherical harmonic basis using numerical experiments, using both randomly generated data and synthetic HRTF measurements. At sample locations, the Slepian basis achieves a reconstruction error up to 11 orders of magnitude smaller than the spherical 
harmonic basis. The Slepian basis also achieves a smaller or equal reconstruction error over the whole sphere for synthetic measurements with and without additive noise.

The necessary mathematical background required to understand this work are contained in Section II. The proposed reconstruction method is presented in Section III. In Section IV, we evaluate the proposed scheme against reconstruction in the spherical harmonic basis. Finally, concluding remarks are made in Section V.

\section{NotATION AND PROPERTIES}

In this section, we present the relevant mathematical background for signals defined on the sphere, in particular the HRTF, and their spectral domain representation in both the spherical harmonic and Slepian bases to clarify concepts and notation used in this work.

\section{A. Signals on the Sphere and Spherical Harmonics}

A point on the unit sphere $\mathbb{S}^{2} \triangleq \boldsymbol{u} \in \mathbb{R}^{3}:\|\boldsymbol{u}\|=1$ can be represented in terms of spherical coordinates as $\boldsymbol{u}(\theta, \phi)=$ $(\sin \theta \cos \phi, \sin \theta \sin \phi, \cos \theta)^{\prime} \in \mathbb{R}^{3}$. Where co-latitude $\theta \in$ $[0, \pi]$ is the angle from the positive $z$-axis and longitude $\phi \in$ $[0,2 \pi)$ is the angle from the positive $x$-axis in the $x-y$ plane. The space of square integrable complex functions on $\mathbb{S}^{2}$ of the form $f(\theta, \phi)$ form a Hilbert space, denoted by $L^{2}\left(\mathbb{S}^{2}\right)$, equipped with the inner product [13]

$$
\langle f, g\rangle \triangleq \int_{\mathbb{S}^{2}} f(\theta, \phi) \overline{g(\theta, \phi)} d s,
$$

where $\overline{(\cdot)}$ is the complex conjugate and $d s=\sin \theta d \theta d \phi$ is the differential area element on the sphere. The norm $\|f\| \triangleq$ $\langle f, f\rangle^{1 / 2}$ is induced by the inner product (1); functions with finite induced norm are referred to as signals on the sphere.

Spherical harmonic functions (spherical harmonics for short) are a commonly used set of complete basis functions that are orthonormal over the whole sphere. Complex spherical harmonics $Y_{\ell}^{m}(\theta, \phi)$ for integer degree $\ell \geq 0$ and order $|m| \leq \ell$ are defined as [13], [14]

$$
Y_{\ell}^{m}(\theta, \phi) \triangleq \sqrt{\frac{2 \ell+1}{4 \pi} \frac{(\ell-m) !}{(\ell+m) !}} P_{\ell}^{m}(\cos \theta) e^{i m \phi},
$$

where $P_{\ell}^{m}(\cdot)$ are the associated Legendre functions. Any signal on the sphere can be expanded in terms of spherical harmonic functions as

$$
f(\theta, \phi)=\sum_{\ell=0}^{\infty} \sum_{m=-\ell}^{\ell}(f)_{\ell}^{m} Y_{\ell}^{m}(\theta, \phi),
$$

where $(f)_{\ell}^{m}$ denotes the spherical harmonic coefficient of degree $\ell$ and order $m$, which form the spectral domain representation of the signal and are given by

$$
(f)_{\ell}^{m} \triangleq \int_{\mathbb{S}^{2}} f(\theta, \phi) \overline{Y_{\ell}^{m}(\theta, \phi)} d s
$$

If the signal $f \in L^{2}\left(\mathbb{S}^{2}\right)$ is band-limited at degree $L$, with $(f)_{\ell}^{m}=0 \forall \ell \geq L,|m| \leq \ell$, then the summation over degree $\ell$ in (3) is truncated at $L-1$. The set of all band-limited signals forms a subspace of $L^{2}\left(\mathbb{S}^{2}\right)$, denoted by $\mathcal{H}_{L}$.

\section{B. Slepian Functions}

Another complete set of basis functions which are orthonormal over the whole sphere are the Slepian functions on the sphere [11], [13], [15]. Slepian functions arise as the solution to the problem, first considered in one-dimension by Slepian and Pollak [16], of finding functions that are band-limited and maximally concentrated within a closed region (or spatially limited and optimally concentrated within some band-limit). Here we briefly present the Slepian problem on the sphere and refer the reader to [15] for a more comprehensive review.

To find signals band-limited at degree $L$, that is $g(\theta, \phi) \in$ $\mathcal{H}_{L}$, and spatially concentrated in a closed region on the sphere $R$, we need to maximise the energy concentration ratio

$$
\lambda=\frac{\int_{R}|g(\theta, \phi)|^{2} d s}{\int_{\mathbb{S}^{2}}|g(\theta, \phi)|^{2} d s} .
$$

This leads to the eigenvalue problem [15]

$$
\sum_{\ell^{\prime}=0}^{L-1} \sum_{m^{\prime}=-\ell^{\prime}}^{\ell^{\prime}} D_{\ell, \ell^{\prime}}^{m, m^{\prime}}(g)_{\ell^{\prime}}^{m^{\prime}}=\lambda(g)_{\ell}^{m},
$$

where $(g)_{\ell}^{m}$ are the coefficients of the Slepian functions in the spherical harmonic basis and

$$
D_{\ell, \ell^{\prime}}^{m, m^{\prime}}=\int_{R} Y_{\ell}^{m}(\theta, \phi) Y_{\ell^{\prime}}^{m^{\prime}}(\theta, \phi) d s
$$

The solution to the eigenvalue problem in (6) gives $L^{2}$ band-limited eigenfunctions which form the Slepian basis. The eigenvalue $0<\lambda<1$ associated with each eigenfunction is a measure of the concentration of the eigenfunction in $R$. The largest eigenvalue $\lambda_{1}$ corresponds to the eigenfunction $g_{1}(\theta, \phi)$ that is most concentrated in $R$, while the smallest eigenvalue $\lambda_{L^{2}}$ corresponds to the eigenfunction $g_{L^{2}}(\theta, \phi)$ that is most concentrated in $\bar{R}$, where $\bar{R}$ is the complement region on the sphere $\bar{R}=\mathbb{S}^{2} \backslash R$. The eigenvalues tend to be either close to zero or one (either concentrated in $R$ or $\bar{R}$ ); finding functions maximally concentrated in $\bar{R}$ is equivalent to solving (6) and reversing the order of eigenfunctions. For the case where $R$ is azimuthal symmetric, $D_{\ell, \ell^{\prime}}^{m, m^{\prime}}$ has an analytical expression and can be computed exactly [10].

Slepian functions have several desirable properties; not only are the orthonormal over the whole sphere, they are also orthogonal over the region $R$, that is

$$
\begin{gathered}
\int_{\mathbb{S}^{2}} g_{\alpha}(\theta, \phi) \overline{g_{\beta}(\theta, \phi)} d s=\delta_{\alpha \beta}, \\
\int_{R} g_{\alpha}(\theta, \phi) \overline{g_{\beta}(\theta, \phi)} d s=\lambda_{\alpha} \delta_{\alpha \beta} .
\end{gathered}
$$

Spherical harmonics, in comparison, are not orthogonal over $R$. As the Slepian basis is complete, for $f(\theta, \phi) \in \mathcal{H}_{L}$ [15], 
$f(\theta, \phi)$ can be expanded in terms of Slepian functions as

$$
f(\theta, \phi)=\sum_{n=1}^{L^{2}}(f)_{n} g_{n}(\theta, \phi),
$$

where $(f)_{n}$ denotes the Slepian coefficients given by

$$
(f)_{n} \triangleq \int_{\mathbb{S}^{2}} f(\theta, \phi) \overline{g_{n}(\theta, \phi)} d s,
$$

and $g_{n}(\theta, \phi)$ is the Slepian function corresponding to eigenvalue $\lambda_{n}$. Over the region $R, f(\theta, \phi)$ can be well-approximated as the summation of only the Slepian functions with $\lambda \approx 1$ [15] with

$$
f(\theta, \phi) \approx \sum_{n=1}^{N}(f)_{n} g_{n}(\theta, \phi),
$$

where $N$ denotes the number of eigenfunctions optimally concentrated in $R$. This enables a more sparse representation of $f(\theta, \phi)$.

\section{Head Related Transfer Function}

The HRTF at a fixed distance from the listener and wavenumber $k=2 \pi f / c$, denoted by $h(\theta, \phi ; k)$ where $c$ is the speed of sound [5], is a signal on the sphere. $h(\theta, \phi ; k)$ is commonly expanded in terms of spherical harmonics [7], [8] and is assumed to be band-limited at degree $L(k)$ with

$$
h(\theta, \phi ; k)=\sum_{\ell=0}^{L(k)-1} \sum_{m=-\ell}^{\ell}(h)_{\ell}^{m}(k) Y_{\ell}^{m}(\theta, \phi) .
$$

The following equation is used to approximate the band-limit $L(k)$ for a particular wavenumber (frequency) [5], [7]

$$
L(k) \approx\left\lceil\frac{e k s}{2}\right\rceil+1=\left\lceil\frac{e \pi s f}{c}\right\rceil+1,
$$

where $s$ is the scattering object size (typically taken to be the radius of the human head). The largest band-limit, corresponding to the maximum audible frequency $f=20 \mathrm{kHz}$ (with $s=0.09 \mathrm{~m}$ ), is $L(k)=47$ [5], [7].

The spherical harmonic coefficients $(h)_{\ell}^{m}(k)$ can be calculated from a finite number of measurements $M$ by writing (13) as a system of linear equations [8]. For a particular wavenumber $k^{1}$

$$
\boldsymbol{h}^{M}=\mathbf{Y}_{L}^{M} \boldsymbol{h}_{L}
$$

where $\mathbf{Y}_{L}^{M}$ is the $M \times L^{2}$-dimensional matrix containing spherical harmonic functions evaluated at the sample locations

$$
\mathbf{Y}_{L}^{M} \triangleq\left(\begin{array}{cccc}
Y_{0}^{0}\left(\theta_{1}, \phi_{1}\right) & Y_{1}^{-1}\left(\theta_{1}, \phi_{1}\right) & \cdots & Y_{L-1}^{L-1}\left(\theta_{1}, \phi_{1}\right) \\
Y_{0}^{0}\left(\theta_{2}, \phi_{2}\right) & Y_{1}^{-1}\left(\theta_{2}, \phi_{2}\right) & \cdots & Y_{L-1}^{L-1}\left(\theta_{2}, \phi_{2}\right) \\
\vdots & \vdots & \ddots & \vdots \\
Y_{0}^{0}\left(\theta_{M}, \phi_{M}\right) & Y_{1}^{-1}\left(\theta_{M}, \phi_{M}\right) & \cdots & Y_{L-1}^{L-1}\left(\theta_{M}, \phi_{M}\right)
\end{array}\right),
$$

\footnotetext{
${ }^{1}$ For the rest of the paper we drop the reference to wavenumber $k$ and simply write $L(k) \equiv L$.
}

where $\boldsymbol{h}_{L}$ is the vector of length $L^{2}$ containing the HRTF coefficients up to degree $L-1$

$$
\boldsymbol{h}_{L}=\left[(h)_{0}^{0},(h)_{1}^{-1}, \ldots,(h)_{L-1}^{L-1}\right]^{T},
$$

and measurements are contained in a vector $\boldsymbol{h}^{M}$ of length $M$,

$$
\boldsymbol{h}^{M}=\left[h\left(\theta_{1}, \phi_{1}\right), h\left(\theta_{2}, \phi_{2}\right), \ldots, h\left(\theta_{M}, \phi_{M}\right)\right]^{T} .
$$

The spherical harmonic coefficients $(h)_{\ell}^{m}(k)$ contained in $\boldsymbol{h}_{L}$ can then be found by inverting $\mathbf{Y}_{L}^{M}$ in (15). This requires at least $M \geq L^{2}$ samples which is the number of spherical harmonic coefficients and the number of degrees of freedom in the system of linear equations. In addition, the sample locations should be chosen such that $\mathbf{Y}_{L}^{M}$ is well-conditioned. The HRTF can then be reconstructed at any location on the sphere from it coefficients using (13).

\section{Proposed HRTF Reconstruction Method}

We propose the reconstruction of the HRTF from its measurements using the Slepian, rather than spherical harmonic, basis. The HRTF can be expanded in the Slepian basis as

$$
h(\theta, \phi ; k)=\sum_{n=1}^{L^{2}}(h)_{n} g_{n}(\theta, \phi),
$$

where $(h)_{n}$ are the Slepian coefficients of the HRTF. (19) can be written as a system of linear equations with

$$
\boldsymbol{h}^{M}=\mathbf{G}_{L}^{M} \widetilde{\boldsymbol{h}}_{L}
$$

where $\mathbf{G}_{L}^{M}$ is the $M \times L^{2}$-dimensional matrix containing Slepian functions evaluated at the $M$ sample locations

$$
\mathbf{G}_{L}^{M} \triangleq\left(\begin{array}{cccc}
g_{1}\left(\theta_{1}, \phi_{1}\right) & g_{2}\left(\theta_{1}, \phi_{1}\right) & \cdots & g_{L^{2}}\left(\theta_{1}, \phi_{1}\right) \\
g_{1}\left(\theta_{2}, \phi_{2}\right) & g_{2}\left(\theta_{2}, \phi_{2}\right) & \cdots & g_{L^{2}}\left(\theta_{2}, \phi_{2}\right) \\
\vdots & \vdots & \ddots & \vdots \\
g_{1}\left(\theta_{M}, \phi_{M}\right) & g_{2}\left(\theta_{M}, \phi_{M}\right) & \cdots & g_{L^{2}}\left(\theta_{M}, \phi_{M}\right)
\end{array}\right),
$$

and $\widetilde{\boldsymbol{h}}_{L}$ is the vector of length $L^{2}$ containing the Slepian coefficients

$$
\widetilde{\boldsymbol{h}}_{L}=\left[(h)_{1},(h)_{2}, \ldots,(h)_{L^{2}}\right]^{T} .
$$

In order to construct $\mathbf{G}_{L}^{M}$, the Slepian functions must be calculated and then evaluated at the $M$ sample locations. For the case where HRTF measurements are not available over the south polar cap region of the sphere, the region of concentration $R$ is the part of the sphere where $\theta<\theta_{c}$, where $\theta_{c}$ is the maximum co-latitude that measurements can be obtained at; typically $\theta_{c}=0.8 \pi$ radians that is $R \triangleq\{0 \leq$ $\left.\theta \leq \theta_{c}, 0 \leq \phi<2 \pi\right\} \subset \mathbb{S}^{2}$. We find the Slepian functions for the region $R$ and for band-limit $L$ by solving the eigenfunction equation $(6)^{2}$. Finally, the Slepian coefficients can be found by inverting $\mathbf{G}_{L}^{M}$, from which the HRTF can be reconstructed by expansion in the Slepian basis.

We summarise the proposed reconstruction method :

${ }^{2} \mathrm{We}$ use matlab code written by Simons et al. available at https://zenodo.org/record/15704\#.VZEHJfmqqko. 
1) Obtain $M$ measurements of the HRTF on the sphere and store these in the vector $\boldsymbol{h}^{M}$.

2) Calculate Slepian functions $g_{n}(\theta, \phi)$ for the region $R$ and the band-limit $L$ for the HRTF wavenumber (frequency) being analysed.

3) Create the matrix $\mathbf{G}_{L}^{M}$ (21) by evaluating $g_{n}(\theta, \phi)$ at the $M$ sample locations.

4) Calculate the HRTF coefficients in the Slepian basis $(h)_{n}$ by solving the system of linear equations (20).

5) From $(h)_{n}$, the HRTF $h(\theta, \phi ; k)$ can be reconstructed over the whole sphere by expansion in the Slepian basis using (19).

\section{A. Sampling Scheme}

Here we discuss the location of the $M$ measurements; the system of linear equations (20) can be solved accurately provided that the number of samples is $M \geq L^{2}$ and $\mathbf{G}_{L}^{M}$ is wellconditioned. It is desirable that the number of measurements taken be as small as possible in order to reduce the time and cost of acquiring the samples [7]; we therefore tested some of the existing sampling schemes on the sphere, that permit samples not being taken over $\theta>\theta_{c}$ and only require $L^{2}$ samples, for reconstruction of the HRTF in both the spherical harmonic and Slepian bases. The reconstruction accuracy was better using the Slepian basis for all schemes and the highest accuracy was obtained, for both the Slepian and SH basis, using the scheme recently proposed in [17]. The sampling scheme [17] is designed to minimise the condition number of matrices composed of sampled spherical harmonics. Designing a sampling scheme which minimises the condition number of a matrix composed of sampled Slepian function is an open problem and should improve the reconstruction accuracy using the Slepian basis further.

The HRTF sampling scheme recently proposed in [17] denoted by $\mathfrak{S}(L)$, is composed of $L$ iso-latitude rings of samples with $2 i+1$ samples in each ring, where $i \in[0, L-1]$ is the ring index. Rings of samples are then arranged in the region $R$ so that the condition number of the matrix to be inverted in the calculation of the spherical harmonic coefficients is as small as possible. An example of this scheme for a band-limit of $L=8$ is shown in Fig. 1 .

\section{Evaluation of Proposed Reconstruction METHOD}

Here we evaluate the accuracy of the proposed method presented in Section III of using the Slepian basis, rather than the spherical harmonic basis, for the reconstruction of the HRTF on the sphere, with measurements obtained using the sampling scheme $\mathfrak{S}(L)$ with $R \triangleq\left\{0 \leq \theta \leq \theta_{c}, 0 \leq \phi<2 \pi\right\}$. First we perform a numerical experiment on randomly generated data to determine the reconstruction accuracy of both bases at sample locations. Then we use a synthetic HRTF model as a ground truth to compare the reconstruction accuracy over the whole sphere for noise free measurements and measurements corrupted by noise. We use the spherical head model [18] to obtain synthetic HRTF data with and without noise added

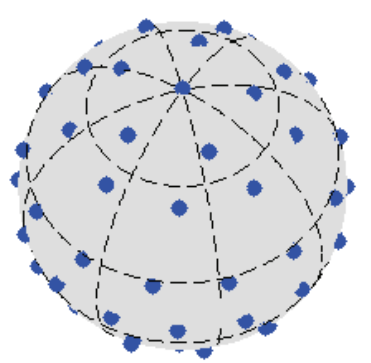

(a)

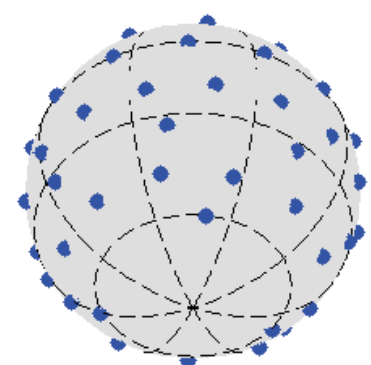

(b)
Fig. 1: Sampling arrangement proposed in [17] for measuring a signal on the sphere band-limited at $L=8$ over the region $\theta \leq \theta_{c}, \theta_{c}=0.8 \pi$ a) north pole view and $b$ ) south pole view.

with the following parameters: head radius $a=0.09 \mathrm{~m}$ with measurements taken over a sphere at a distance of $r=1$ $\mathrm{m}$ from the head. This model also requires a threshold level at which the spherical Hankel functions are calculated to be specified; we set the threshold at $10^{-15}$ (machine precision).

\section{A. Analysis of Reconstruction at Sample Locations}

We first compare the reconstruction accuracy obtained using the Slepian and spherical harmonic bases on a randomly generated band-limited signal. The purpose of this experiment is to compare the reconstruction accuracy when there is no truncation error present (although the HRTF is assumed to be band-limited there is actually a small amount of energy present at higher frequencies [5], [17]).

We carry out the following experiment for band-limits in the range $8 \leq L \leq 64$ : a complex valued band-limited test signal $f_{\mathrm{T}} \in \mathcal{H}_{L}$ with real and imaginary parts randomly chosen from a uniform distribution on the interval $[-1,1]$ is generated over $\mathfrak{S}(L)$. The signal is then reconstructed in the Slepian basis, adopting the method described in Section III, or in the spherical harmonic basis, as described in Section II-C, to obtain the reconstructed signal $f_{\mathrm{R}}$ on $\mathfrak{S}(L)$. The maximum reconstruction error $E_{\max }$ and mean reconstruction error $E_{\text {mean }}$ taken over the sample locations $\mathfrak{S}(L)$ are defined as

$$
\begin{aligned}
& E_{\max } \triangleq \max \left|f_{\mathrm{T}}(\theta, \phi)-f_{\mathrm{R}}(\theta, \phi)\right|, \\
& E_{\text {mean }} \triangleq \frac{1}{L^{2}} \sum_{(\theta, \phi)}\left|f_{\mathrm{T}}(\theta, \phi)-f_{\mathrm{R}}(\theta, \phi)\right| .
\end{aligned}
$$

We repeat this experiment 10 times and calculate the average values for the maximum and mean error between the original and reconstructed signal.

Fig. 2 shows the maximum and mean reconstruction error, $E_{\text {max }}$ and $E_{\text {mean }}$, obtained using the Slepian and spherical harmonic bases. The reconstruction errors $E_{\max }$ and $E_{\text {mean }}$ in the Slepian basis is much smaller than in the spherical harmonic basis for all band-limits $8 \leq L \leq 64$. Reconstruction of $f_{\mathrm{T}}$ in the Slepian basis is up to 11 orders of magnitude more accurate than in the spherical harmonic basis; at band-limit $L=48$, (corresponding to the maximum audible frequency of $f=20 \mathrm{kHz}$ ) the Slepian basis has $E_{\text {mean }}$ on the order of $10^{-14}$, 


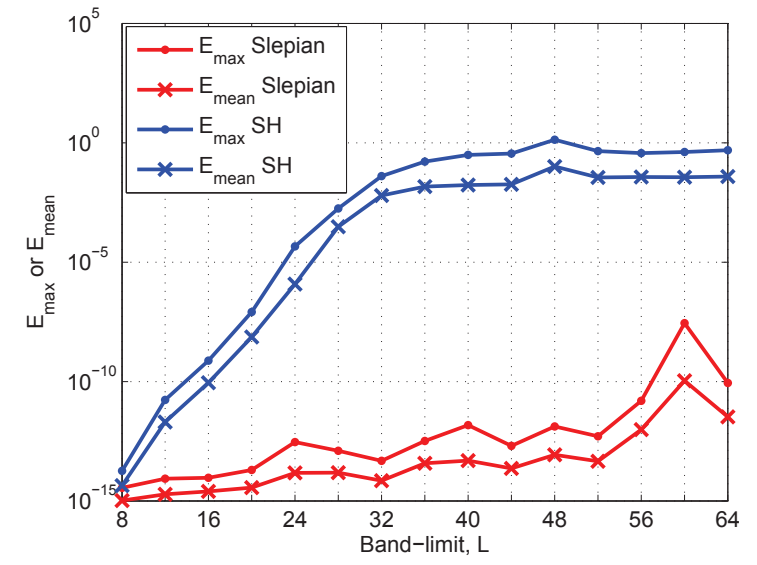

Fig. 2: Reconstruction error at sample locations: plots of the maximum error $E_{\max }$ and the mean error $E_{\text {mean }}$, respectively given in (23) and (24), obtained using the Slepian and spherical harmonic $(\mathrm{SH})$ basis for band-limits $8 \leq L \leq 64$.

while $E_{\text {mean }}$ is on the order of $10^{-3}$ for the spherical harmonic basis. The difference in reconstruction error is related to the spherical harmonics not being a natural basis for the region $R$, whereas the Slepian functions are designed based on $R$ and are orthogonal over $R$.

\section{B. HRTF Reconstruction Analysis}

In order to evaluate the numerical accuracy of the proposed scheme in the reconstruction of the HRTF over the whole sphere, not just at locations where measurements have been obtained, we conduct the following experiment. For a given frequency $f$ (corresponding to wavenumber $k$ ), synthetic measurements of the HRTF $h(\theta, \phi ; k)$ are obtained from the spherical head model [18] over $\mathfrak{S}(L)$. The HRTF is the reconstructed over a high resolution equiangular grid (consisting of 197192 points) in the Slepian (Section III) and spherical harmonic (Section II-C) bases . For both the HRTF signal reconstructed using the Slepian basis and the HRTF reconstructed using the spherical harmonic basis, we compute the error $E_{R}(\theta, \phi ; k)$ between the reconstructed $\operatorname{HRTF} h_{R}(\theta, \phi ; k)$ and the analytical value of the HRTF $h_{A}(\theta, \phi ; k)$ obtained from the spherical head model, given by

$$
E_{R}(\theta, \phi ; k) \triangleq\left|h_{A}(\theta, \phi ; k)-h_{R}(\theta, \phi ; k)\right| .
$$

When this experiment is performed on synthetic HRTF data with band-limit $L(k)$ calculated using the formula (14); the same reconstruction error is obtained using the Slepian or spherical harmonic basis. It was shown in [17] that there is significant energy in the spherical head model HRTF for spherical harmonic degrees above the band-limit given by this formula. Increasing the band-limit of the HRTF reduces the truncation error, allowing for a difference in the reconstruction error $E_{R}(\theta, \phi ; k)$ to be observed between the two bases.

The reconstruction error $E_{R}(\theta, \phi ; k)$ for an audible frequency of $f=5 \mathrm{kHz}$ and band-limit $L=28$ (as was used in

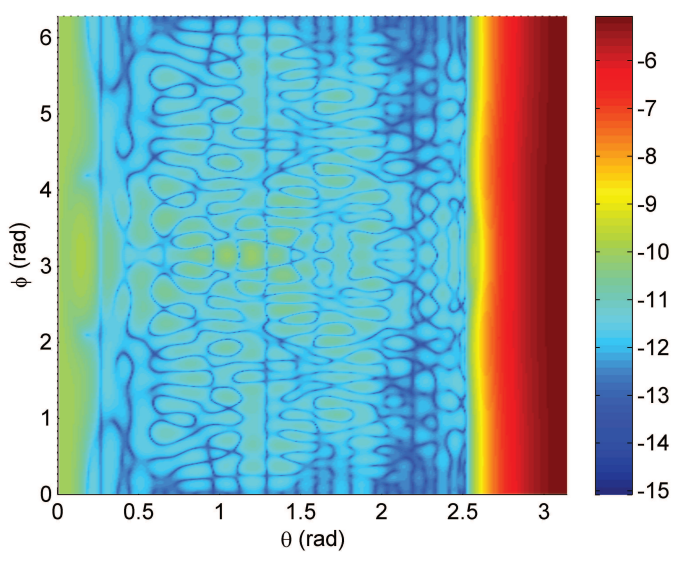

(a)

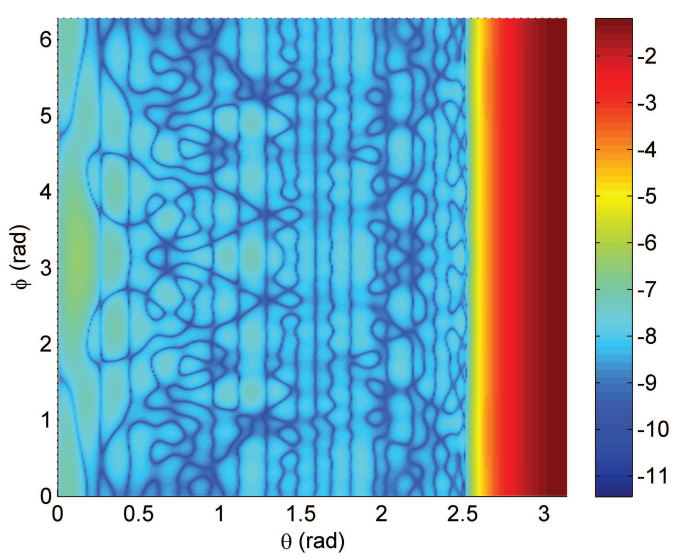

(b)

Fig. 3: Reconstruction error over the whole sphere: $E_{R}(\theta, \phi ; k)$, given by (25), of the HRTF on the sphere at $f=5$ $\mathrm{kHz}$ using a band-limit of $L=28$ for the a) Slepian and b) spherical harmonics basis.

[17]) is plotted in Fig. 3(a) for the Slepian basis and Fig. 3(b) for the spherical harmonic basis. $E_{R}(\theta, \phi ; k)$ is significantly smaller at all locations on the sphere, both inside and outside of the sampled region $R$, for $h_{R}(\theta, \phi ; k)$ reconstructed in the Slepian basis compared with reconstruction in the spherical harmonic basis. The error $E_{R}(\theta, \phi ; k)$ is also plotted for of $f=15 \mathrm{kHz}$ and $L=47$ in Fig. 4(a) for the Slepian basis and Fig. 4(b) for the spherical harmonic basis. $E_{R}(\theta, \phi ; k)$ is significantly smaller in the region $R$ for the Slepian basis and similar in $\bar{R}$ for reconstruction in both basis.

\section{Noisy HRTF Reconstruction Analysis}

It is important to evaluate if the Slepian basis allows for robust reconstruction in the presence of noise; here we perform preliminary investigations into using the Slepian basis for reconstruction when additive noise is present. In geophysics, the Slepian basis is truncated as a means of filtering the noise [10]; we apply this method to HRTF measurements.

To study the effect of noise, we carry out a following 


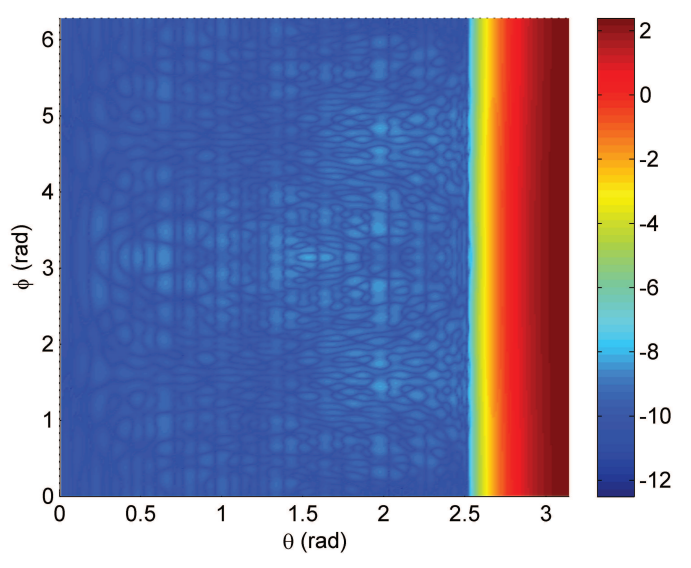

(a)

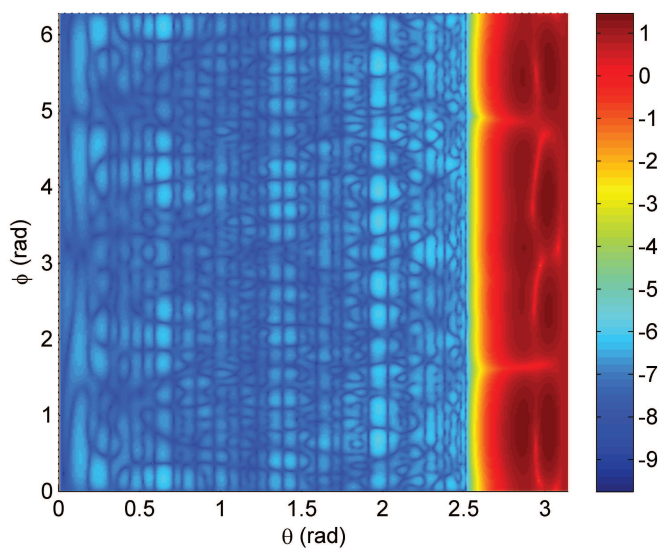

(b)

Fig. 4: Reconstruction error over the whole sphere: $E_{R}(\theta, \phi ; k)$, given by (25), of the HRTF on the sphere at $f=15 \mathrm{kHz}$ using a band-limit of $L=47$ for the a) Slepian and b) spherical harmonics basis.

experiment: samples of the HRTF $h(\theta, \phi ; k)$, given by the spherical head model and taken over the audible frequency range with complex Gaussian noise of a constant variance added to each measurement, are obtained using $\mathfrak{S}(L)$. The samples containing noise $h_{N}(\theta, \phi ; k)$ are then used reconstruct the HRTF, in both the Slepian and spherical harmonic bases, on a high resolution equiangular grid (197192 points) $h_{R}(\theta, \phi ; k)$. The reconstruction error $E_{R}(\theta, \phi ; k)$ given in (25) between the reconstructed HRTF $h_{R}(\theta, \phi ; k)$ and the analytical value of the HRTF (without noise) $h_{A}(\theta, \phi ; k)$ obtained from the spherical head model is then calculated for reconstruction in both the Slepian and spherical harmonic basis.

We show results for a small amount of noise added to measurements (with variance corresponding to a signal-tonoise ration of $40 \mathrm{~dB}$ ). Reconstruction in the Slepian and spherical harmonics gives very similar results for $E_{R}(\theta, \phi ; k)$. We truncate the Slepian basis in order to reduce $E_{R}(\theta, \phi ; k)$; we present results with only a small degree of truncation (with the 10 Slepian functions that are the least concentrated in $R$

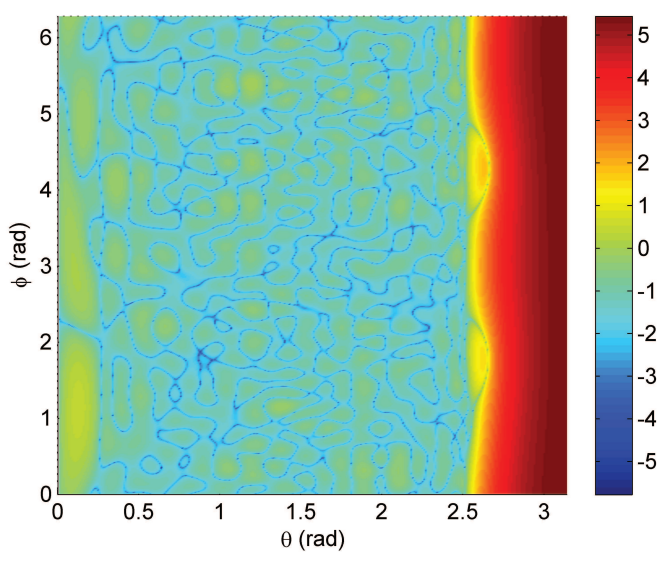

(a)

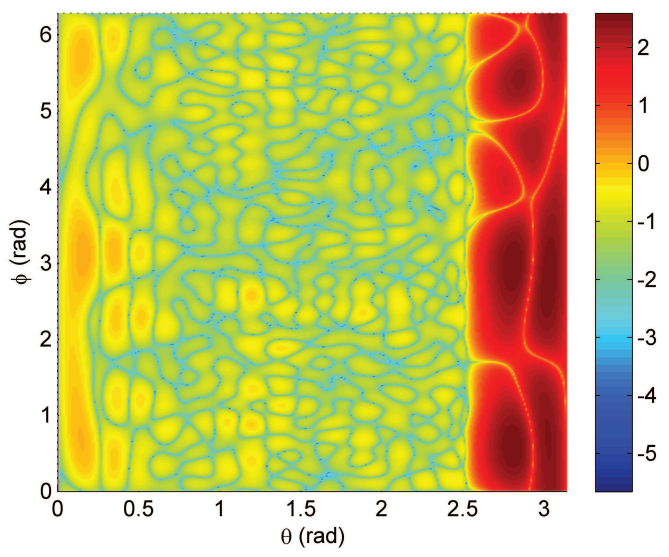

(b)

Fig. 5: Reconstruction error in the presence of noise: $E_{R}(\theta, \phi ; k)$, given by (25), of the HRTF on the sphere at $f=5$ $\mathrm{kHz}$ for band-limit $L=28 \mathrm{kHz}$ a) spherical harmonic and b) truncated Slepian basis.

removed). Choosing an optimal truncation degree based on signal-to-noise ratio and comparing truncation of the Slepian basis to regularised least-squares is considered future work. Fig. 5 shows $E_{R}(\theta, \phi ; k)$ for $f=5 \mathrm{kHz}$ and $L=28$ for the a) spherical harmonic basis and b) truncated Slepian basis. In Fig. $6 E_{R}(\theta, \phi ; k)$ is plotted for $f=15 \mathrm{kHz}$ and $L=47$ for the a) spherical harmonic basis and b) truncated Slepian basis. $E_{R}(\theta, \phi ; k)$ is slightly smaller inside of $R$ and much smaller in $\bar{R}$ for the truncated Slepian basis compared with the spherical harmonic (and full Slepian basis) for both $f=5$ $\mathrm{kHz}$ and $f=15 \mathrm{kHz}$. This is because the 10 least concentrated Slepian functions in the region $R$ contribute the least to $h(\theta, \phi)$ inside $R$ and the contribute the most in $\bar{R}$.

Our numerical analysis in this section shows better or similar reconstruction performance obtained using the Slepian basis for HRTF reconstruction, rather than the spherical harmonic basis, over the whole sphere; at sample locations, in the region $R$ and in the polar cap $\bar{R}$. 


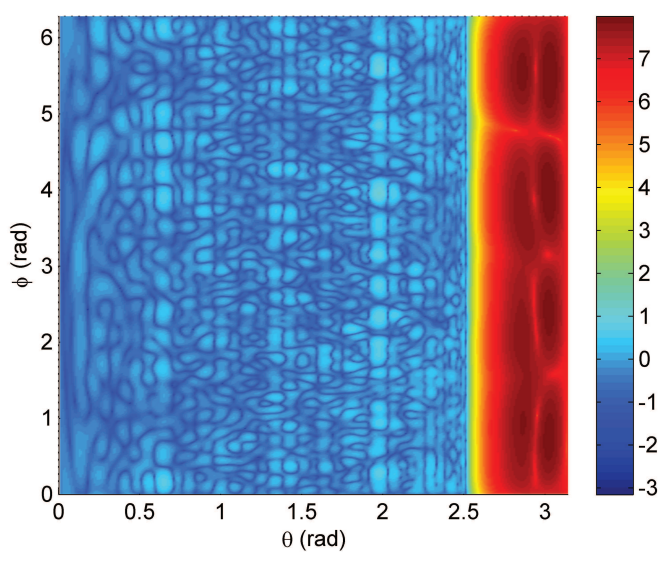

(a)

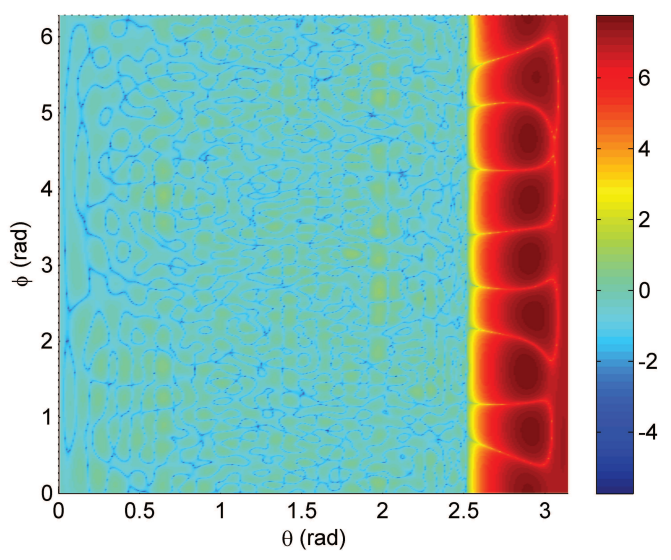

(b)

Fig. 6: Reconstruction error in the presence of noise: $E_{R}(\theta, \phi ; k)$, given by (25), of the HRTF on the sphere at $f=15 \mathrm{kHz}$ for band-limit $L=47$ a) spherical harmonic and b) truncated Slepian basis.

\section{Conclusions}

In this work, we have investigated the use of Slepian functions for the reconstruction the HRTF on the sphere when measurements are unavailable from the south polar cap, rather than the traditionally used spherical harmonics. While spherical harmonics are well suited to applications where data is available over the whole sphere, they are not a natural basis for modeling a region of the sphere. Slepian functions on the other hand, are designed for the region and are not only orthonormal over the whole sphere but orthogonal over the region. We proposed a method of reconstructing the HRTF using the Slepian basis. We demonstrated through numerical experiments, on randomly generated data and synthetic HRTF measurements, that reconstruction of the HRTF in the Slepian basis is significantly more accurate at sample locations as well as other locations on the sphere, both inside and outside the region where measurements were obtained, than in the spherical harmonic basis. We also briefly investigated truncation of the Slepian basis when reconstruction was carried out on noisy HRTF measurements, which we found decreased the reconstruction error. Further investigation should be carried out into finding an optimal truncation degree depending on the signal-to-noise ratio and comparing the truncation in the Slepian basis to regularised least-squares in the spherical harmonic basis. From this investigation, we found that the Slepian basis allows for more accurate reconstruction of the HRTF than the spherical harmonic basis, though further investigation into the open questions we have discussed is necessary.

\section{REFERENCES}

[1] C. D. Salvador Castaneda, S. Sakamoto, J. A. Trevino Lopez, J. Li, Y. Yan, and Y. Suzuki, "Accuracy of head-related transfer functions synthesized with spherical microphone arrays," in Proc. Mtgs. Acoust., vol. 19, no. 1, Montreal, Canada, Jun. 2013.

[2] Q. Huang and Y. Fang, "Interpolation of head-related transfer functions using spherical fourier expansion," Chinese J. Electron., vol. 26, no. 4 , pp. 571-576, Jul. 2009.

[3] V. R. Algazi, R. O. Duda, D. M. Thompson, and C. Avendano, "The CIPIC HRTF database," in Proc. IEEE Workshop Applicat. Signal Process. Audio and Acoust., New Paltz, NY, 2001, pp. 99-102.

4] Z. Li and R. Duraiswami, "Flexible and optimal design of spherical microphone arrays for beamforming," IEEE Trans. Acoust., Lang. Signal Process., vol. 15, no. 2, pp. 702-714, Feb. 2007.

[5] W. Zhang, T. D. Abhayapala, R. A. Kennedy, and R. Duraiswami, "Insights into head-related transfer function: Spatial dimensionality and continuous representation," J. Acoust. Soc. Am., vol. 127, no. 4, pp. 2347-2357, Apr. 2010.

[6] Y. Luo, D. N. Zotkin, H. Daume, and R. Duraiswami, "Kernel regression for head-related transfer function interpolation and spectral extrema extraction," in Proc. IEEE Int. Conf. Acoust., Speech, Signal Process., ICASSP'2013, Vancover, Canada, May 2013, pp. 256-260.

[7] W. Zhang, M. Zhang, R. A. Kennedy, and T. D. Abhayapala, "On highresolution head-related transfer function measurements: An efficient sampling scheme," IEEE Trans. Acoust., Lang., Signal Process., vol. 20, no. 2, pp. 575-584, Feb. 2012.

[8] D. N. Zotkin, R. Duraiswami, and N. A. Gumerov, "Regularized HRTF fitting using spherical harmonics," in Proc. IEEE Workshop Applicat. Signal Process. Audio and Acoust., New Paltz, NY, Oct. 2009, pp. 257 260.

[9] M. J. Evans, J. A. S. Angus, and A. I. Tew, "Analyzing head-related transfer function measurements using surface spherical harmonics," $J$. Acoust. Soc. Am., vol. 104, no. 4, pp. 2400-2411, Jun. 1998.

[10] F. J. Simons and F. A. Dahlen, "Spherical slepian functions and the polar gap in geodesy," Geophys. J. Int., vol. 166, no. 3, pp. 1039-1061, 2006.

[11] A. Albertella, F. Sansó, and N. Sneeuw, "Band-limited functions on a bounded spherical domain: the Slepian problem on the sphere," $J$. Geodesy, vol. 73, no. 9, pp. 436-447, 1999.

[12] N. Sneeuw and M. van Gelderen, "The polar gap," in Geodetic Boundary Value Problems in View of the One Centimeter Geoid, ser. Lecture Notes in Earth Sciences, F. Sansó and R. Rummel, Eds. Springer Berlin Heidelberg, 1997, vol. 65, pp. 559-568.

[13] R. A. Kennedy and P. Sadeghi, Hilbert Space Methods in Signal Processing. Cambridge, UK: Cambridge University Press, Mar. 2013.

[14] J. J. Sakurai, Modern Quantum Mechanics, 2nd ed. Reading, MA: Addison Wesley Publishing Company, Inc., 1994.

[15] F. J. Simons, F. A. Dahlen, and M. A. Wieczorek, "Spatiospectral concentration on a sphere," SIAM Review, vol. 48, no. 3, pp. 504-536, 2006.

[16] D. Slepian and H. O. Pollak, "Prolate spheroidal wave functions, fourier analysis and uncertainty i," Bell Syst. Tech. J., vol. 40, no. 1, pp. 43-63, 1961

[17] A. P. Bates, Z. Khalid, and R. A. Kennedy, "Novel sampling scheme on the sphere for head-related transfer function measurements," IEEE Trans. Acoust., Lang., Signal Process., vol. 23, no. 6, pp. 1068-1082, Jun. 2015.

[18] R. O. Duda and W. L. Martens, "Range dependence of the response of a spherical head model," J. Acoust. Soc. Am., vol. 104, no. 5, pp. 3048-3058, Nov. 1998. 